\title{
Magnetooptic Effects in Surface-Plasmon-Polaritons Slab Waveguides
}

\author{
B. Sepúlveda, L. M. Lechuga, and G. Armelles
}

\begin{abstract}
This paper analyzes the magnetooptic (MO) effects in surface plasmon polaritons (SPP) propagating in thin metallic layers as a function of the metal thickness. Two different configurations are analyzed: a ferromagnetic metal surrounded by nonmagnetic dielectrics and a nonmagnetic metal bounded by ferromagnetic dielectrics. Depending on the configuration and the thickness of the metallic layer, waveguides with very low attenuation losses and high MO effects can be obtained. These configurations could be used to design MO active devices in SPP-based optics.
\end{abstract}

Index Terms-Isolators, magnetooptic (MO) effects, magnetooptic (MO) waveguides, nonreciprocal waveguides, surface plasmon polariton (SPP), surface plasmon polariton (SPP) waveguides.

\section{INTRODUCTION}

$\mathbf{T}$ HE REDUCTION of the dimensions of the conventional optical components in the way to the nanooptic is restricted by the diffraction limit of the light. However, this barrier can be overcome with the surface plasmon polariton (SPP)-based optic. The surface plasmons (SPs) are collective oscillations of the metal electron gas that propagate at the interface of a metal and a dielectric. Such oscillations generate highly confined electromagnetic fields whose maximum amplitude is located at the interface and decays exponentially at both sides of the interface. Therefore, thin films of noble metals can be used as waveguides for the SP. Besides, the nanoscale modification of the metal layers can lead to the manipulation and control of the SP in the submicrometer range [1]. Within this context, experimental and theoretical works describing two-dimensional (2-D) SPP confinement in strip waveguides [2]-[7] and mirrors, divisors or interferometers with 2-D confinement have been reported recently [8], [9]. These components are the basis for the development of very attractive passive nanooptic devices. In a next stage, it would be desirable to achieve active components to control the propagation properties of the SPP, like the ones that exist in the conventional integrated optics as switches, modulators, or isolators. Such devices could be based on magnetooptic (MO) effects. However, there is little work concerned with the study of the MO properties in the propagation of SPP up to date. For example, the MO effects of surface plasmons in semi-infinite media has been

Manuscript received November 19, 2004; revised July 14, 2005. This work was supported by the Ministerio de Ciencia y Tecnología under Project MAT2002-04484-C03-01. The work of B. Sepúlveda is supported by the Consejo Superior de Investigaciones Científicas (CSIC) under the I3P program. The authors are with Instituto de Microelectrónica de Madrid (CNM-CSIC), Madrid 28760, Spain (e-mail: borja@imm.cnm.csic.es).

Digital Object Identifier 10.1109/JLT.2005.861943

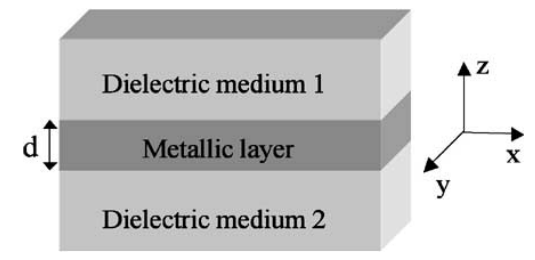

Fig. 1. Structure of the layers in an SPP waveguide.

treated in [10] and [11]; the dispersion relation of magnetoplasma modes in thin films in the nonretarded or very thin film limits without losses has been studied in [12]-[14]; the enhancement of the MO Kerr effects in multilayers of noble and ferromagnetic metals have been reported in [15]-[17]; or the effect of the application of a magnetic field in the extraordinary transmission through a nanostructured metal film has been described in [18]. Here, we analyze the propagation properties of SPPs guided by lossy metallic layers for different orientations of the magnetization as a function of the thickness of the metallic layer, without making any approximation in the calculation of the dispersion relation and field distribution of the SPP modes. In this study, two configurations are considered: a nonmagnetic metallic layer bounded by a ferromagnetic dielectric material like the yttrium iron garnets (YIGs) and a ferromagnetic metallic layer like cobalt surrounded by nonmagnetic dielectric media. Ferrimagnetic YIGs have attracted great interest in the integrated optics field due to their high transparency at communication wavelengths. In addition, YIGs are soft magnetic materials showing in-plane magnetization and very low saturation magnetic field, below 50 Oe [19], making their technological application more feasible. On the other hand, ferromagnetic metals show stronger attenuation losses than the metals typically used in plasmonic applications, limiting its use within this field. Regarding the magnetic properties, polycrystalline thin cobalt layers also exhibit in-plane magnetization and low saturation magnetic field, around 50 Oe.

The paper is organized as follows. Section II briefly introduces the optical properties of the SPP in the isotropic media and describes the theoretical formalisms used in the MO calculations. Meanwhile, Section III shows the calculations of the MO effects for the different orientations of the magnetization. Finally, Section IV will analyze the most interesting configurations more deeply in order to use their properties for technological applications.

\section{TheORETICAL Formalism}

In Fig. 1, we present a schematic of the system under consideration, which is formed by a metallic layer whose thickness 

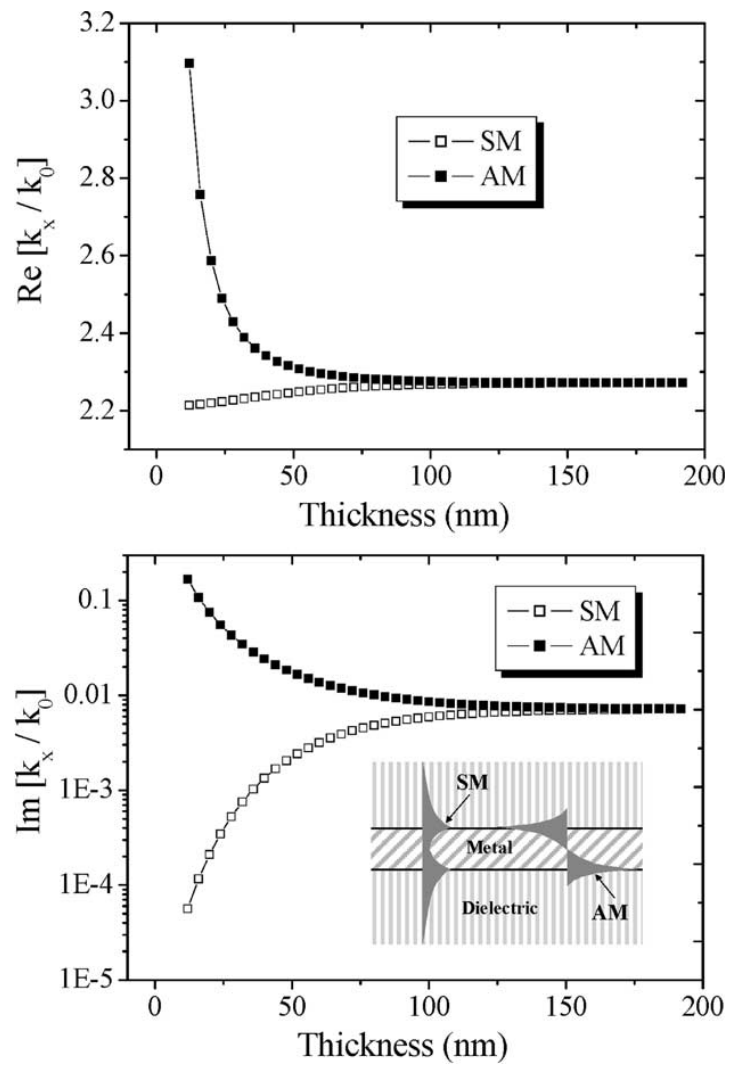

Fig. 2. Real and imaginary parts of the propagation wavevector $k_{x}$ for the SPP modes (SM and AM) in a gold layer surrounded by a dielectric medium with $\varepsilon_{d}=4.84$, as a function of the gold thickness. The inset in the imaginary part of $k_{x}$ displays the field distribution of the $y$ component of the magnetic field $H_{y}$ of the SM and AM.

is given by $d$ and surrounded by two dielectric materials. This metallic layer acts as a waveguide for the SPP, where we assume that the SPP propagation is along the $x$ direction. If the metallic layer is thick enough, two independent bounded surface plasmons can be excited in the metallic layer-one in each metal/dielectric interface. The dispersion relation $\omega\left(k_{x}\right)$ of these surface plasmons is obtained by solving the well-known equation [20]

$$
k_{x}=k_{0} \sqrt{\frac{\varepsilon_{m} \varepsilon_{d i}}{\varepsilon_{m}+\varepsilon_{d i}}}
$$

where $k_{0}=\omega / c, \omega$ denotes the frequency, $c$ denotes the speed of light in vacuum, and $\varepsilon_{d i}$ and $\varepsilon_{m}$ are the dielectric constants of the dielectrics and the metal, respectively. At the same time, the electric and the magnetic fields of the SPP $\vec{E}(\vec{r}, t)$ and $\vec{H}(\vec{r}, t)$ show an exponential decay towards the metal and the dielectric media and a transversal magnetic (TM) polarization.

As the metallic layer thickness decreases, there is an interaction between the surface plasmons of both interfaces [21]-[23]. If the dielectric media at both sides of the metallic layer are the same, the interaction between the SP gives rise to two electromagnetic guided modes: one with a symmetric distribution of the electromagnetic field called the symmetric mode (SM) and another with an antisymmetric field distribution denoted as the antisymmetric mode (AM), as can be seen in the
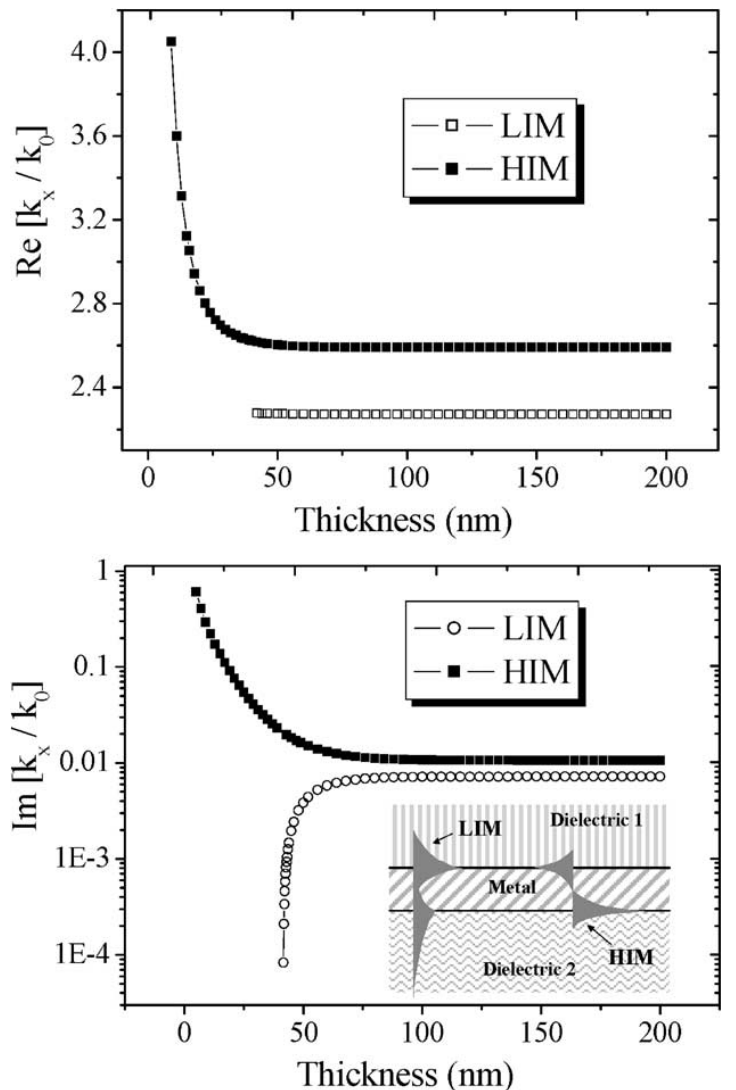

Fig. 3. Real and imaginary parts of the propagation wavevector $k_{x}$ for SPP modes (LIM and HIM) in a gold layer surrounded by a dielectric medium with $\varepsilon_{d 1}=4.84$ at one side and dielectric material with a higher dielectric constant $\varepsilon_{d 2}=6.25$ at the other, as a function of the gold thickness. In this configuration, the cutoff of the LIM is produced at gold thickness of $40 \mathrm{~nm}$. The inset in the imaginary part of $k_{x}$ displays the field distributions of the $y$ component of the magnetic field $H_{y}$ of the LIM and HIM.

inset of Fig. 2. As a result, the electromagnetic field of the SM spreads out in the dielectric media when the thickness of the metallic layer decreases, diminishing strongly its attenuation losses. For that reason, this mode is called the long-range mode. Meanwhile, the AM penetrates deeper into the metal causing an enhancement in its attenuation losses. This is better seen in Fig. 2 where we present the real and the imaginary parts of the propagation wave vector $k_{x}$ as functions of the metallic thickness for a gold layer surrounded by dielectric media. In these calculations, the dielectric constants of gold [24] and the dielectric medium are $\varepsilon_{m}=-90.11+i 10.07$ and $\varepsilon_{d}=4.84$, respectively, at a wavelength of $1500 \mathrm{~nm}$. The selected $\varepsilon_{d}$ is similar to the dielectric constant of the YIG at that wavelength [19]. On the other hand, if the dielectric media have different refractive indexes and the metallic layer is thick enough, the SPP modes at each metal/dielectric interface show different wave vectors and field distributions. When the metallic thickness is reduced, the interaction of both SPP no longer produces symmetric or antisymmetric field distributions. However, there is still a reduction of the attenuation losses for one of the two modes as the thickness of the metallic layer is decreased. This behavior can be seen in Fig. 3, where we present the same case as in Fig. 2; but now, one of the dielectric media has a dielectric constant of $\varepsilon_{d 2}=6.25$, similar to the one of the $\mathrm{TiO}_{2}$ 
or $\mathrm{ZnSe}$ at $1500 \mathrm{~nm}$. These modes can be denoted as the lower index mode (LIM) or the higher index mode (HIM), depending on the location of the maximum amplitude with respect to the dielectric media as the inset of Fig. 3 shows. The electromagnetic field of the LIM extends toward the higher index dielectric medium as the thickness of the metallic layer decreases. Consequently, its attenuation loss is reduced up to the cutoff thickness where the mode is no longer guided. Accordingly, this mode is also called the long-range mode. In opposition, the HIM shows a deeper penetration into the metal and stronger attenuation losses.

Such behavior of the electromagnetic modes with the film thickness is also valid when any of the media present an MO activity. However, the theoretical treatment of these modes is more complicated since the magnetization causes the coupling of the different components of the electromagnetic fields. For example, up to the first order in magnetization, the dielectric tensor of an MO active material with cubic symmetry is given by

$$
\varepsilon=\left(\begin{array}{ccc}
\varepsilon & a M_{z} & a M_{y} \\
-a M_{z} & \varepsilon & -a M_{x} \\
-a M_{y} & a M_{x} & \varepsilon
\end{array}\right)
$$

where $a$ is the MO constant and $M_{i}$ s are the different components of the magnetization. As can be observed in this nonsymmetric dielectric tensor, the nondiagonal elements of the dielectric tensor are responsible for the coupling of the electromagnetic components. Therefore, in general, the transversal electric (TE) and TM polarizations cannot be treated separately. Then, to calculate the dispersion relation of the bounded modes when MO media are involved, the $4 \times 4$ matrix formalism developed by Vassell [25] can be used. This formalism has been employed to calculate the guided modes for arbitrary anisotropic layered dielectric waveguides. Such formalism is based on the continuity of the tangential components of the electromagnetic fields at the interfaces of each layer ( $x$ and $y$ components) and the imposition of an evanescent field behavior at both sides of the layer stack. Accordingly, the calculation of the dispersion relation and the field distribution of the SPP guided modes when MO media are involved can be carried out identically.

Within this formalism, the tangential components at both sides of the multilayer are related by a $4 \times 4$ total transfer matrix, which is calculated through the ordered product of the partial $4 \times 4$ matrix of each layer

$$
\left(\begin{array}{c}
E_{x i} \\
E_{y i} \\
H_{x i} \\
H_{y i}
\end{array}\right)=\left(\begin{array}{llll}
T_{11} & T_{12} & T_{13} & T_{14} \\
T_{21} & T_{22} & T_{23} & T_{24} \\
T_{31} & T_{32} & T_{33} & T_{34} \\
T_{41} & T_{42} & T_{43} & T_{44}
\end{array}\right)\left(\begin{array}{c}
E_{x f} \\
E_{y f} \\
H_{x f} \\
H_{y f}
\end{array}\right)
$$

where the subscripts $i$ and $f$ represent the initial and final media, respectively. The partial transfer matrix of an arbitrary anisotropic layer is obtained through the following equation [26]

$$
T_{p}=\exp \left\{i k_{0} \boldsymbol{\Delta} d\right\}=\beta_{0} \mathbf{I}+\beta_{1} \boldsymbol{\Delta}+\beta_{2} \boldsymbol{\Delta}^{2}+\beta_{3} \boldsymbol{\Delta}^{3}
$$

where $d$ is the thickness of the layer, $\mathbf{I}$ is the identity $4 \times 4$ matrix, and matrix $\Delta$ is given by

$$
\boldsymbol{\Delta}=\left(\begin{array}{cccc}
-k_{x} \frac{\varepsilon_{31}}{\varepsilon_{33}} & -k_{x} \frac{\varepsilon_{32}}{\varepsilon_{33}} & 0 & 1-\frac{k_{x}^{2}}{\varepsilon_{33}} \\
0 & 0 & -1 & 0 \\
\varepsilon_{23} \frac{\varepsilon_{31}}{\varepsilon_{33}}-\varepsilon_{21} & k_{x}^{2}-\varepsilon_{22}+\varepsilon_{23} \frac{\varepsilon_{32}}{\varepsilon_{33}} & 0 & k_{x} \frac{\varepsilon_{23}}{\varepsilon_{33}} \\
\varepsilon_{11}-\varepsilon_{13} \frac{\varepsilon_{31}}{\varepsilon_{33}} & \varepsilon_{12}-\varepsilon_{13} \frac{\varepsilon_{321}}{\varepsilon_{33}} & 0 & -k_{x} \frac{\varepsilon_{13}}{\varepsilon_{33}}
\end{array}\right) .
$$

In this matrix, $\varepsilon_{i j}$ represents the different elements of the dielectric tensor. In addition, the different scalars $\beta_{i}$ must satisfy the following set of equations

$$
\exp \left\{i k_{0} q_{k} d\right\}=\sum_{j=1}^{4} \beta_{j} q_{k}^{j} \quad k=1, \ldots, 4
$$

where $q_{k}$ are the eigenvalues of matrix $\boldsymbol{\Delta}$.

The imposition of the exponential decay in the initial and final media, together with the continuity of the electromagnetic field components parallel to the interfaces of the layer stack, give rise to a homogeneous system of equations. The nontrivial solution of this system generates an equation whose resolution gives the transverse resonant condition, that is, the component of the wave vector of the SPP parallel to the direction of propagation $\left(k_{x}\right)$. Once $k_{x}$ is calculated, the electromagnetic field distribution in each layer can be determined. One advantage of this formalism is that it provides exact analytical solutions that can be used to generate more complicated structures, like stripes, through numerical methods [27]. This formalism will be used in Section III to calculate the dispersion relation and the field distribution of the bounded SPP modes as a function of the metallic film thickness for the different orientations of the magnetization in the MO media.

\section{MO EFFECTS}

\section{A. Transversal Magnetization}

First, let us treat the case of the magnetization in the transversal directions $M_{y}=M$ and $M_{x}=M_{z}=0$. In this configuration of the magnetization, the dielectric tensor is given by

$$
\varepsilon=\left(\begin{array}{ccc}
\varepsilon & 0 & a M \\
0 & \varepsilon & 0 \\
-a M & 0 & \varepsilon
\end{array}\right)
$$

As a consequence, the magnetization couples the $x$ and the $z$ components of the electric fields $E_{x}$ and $E_{z}$, maintaining the independence of the TE and the TM polarizations in the Maxwell equations. Therefore, the SPP guided modes will keep the TM behavior in this configuration.

To understand the MO behavior of the SPP guided modes in a metallic layer, we first consider the case of a single interface separating a metal and a dielectric. We assume that the metal is 
(a)
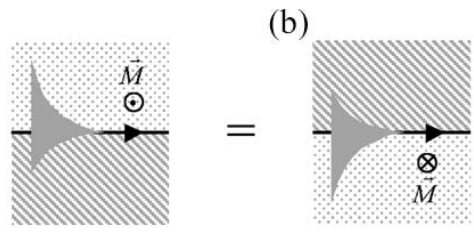

(c)

Dielectric

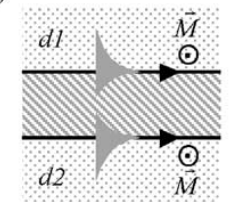

(d)

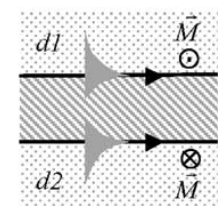

Fig. 4. Equivalence relations between SPP waveguides as a function of the direction of the magnetization of the dielectric layers. The electromagnetic field distribution corresponds to the $y$ component of the magnetic field $H_{y}$.

not magnetic, while the dielectric medium shows MO activity like the ferromagnetic dielectric YIG [see Fig. 4(a)]. In this case, the wave vector of the SPP mode $k_{x}$ is as in (8), shown at the bottom of the page.

In (8), $\varepsilon_{m}$ is the dielectric constant of the metal, $\varepsilon_{d}$ and $a_{d}$ are the dielectric and the MO constants of the dielectric medium, $M$ is the magnetization of the ferromagnetic dielectric, and the \pm sign corresponds to the two possible directions of the magnetization. As can be observed, the wave vector of the surface plasmon of a single interface depends on the direction of the magnetization. Also, the following relation between forward and backward traveling modes is satisfied [28]

$$
k_{x}^{+}( \pm \bar{M})=-k_{x}^{-}(\mp \bar{M})
$$

where the $(+)$ and $(-)$ superscripts represent forward and backward traveling modes, respectively. Therefore, if $k_{x}^{+}(\bar{M}) \neq$ $k_{x}^{+}(-\bar{M})$, then the forward and the backward traveling modes also have different dispersion relations, i.e., the magnetization induces a nonreciprocity in the dispersion relation $\omega\left(k_{x}\right) \neq$ $\omega\left(-k_{x}\right)$.

The interchange of the metallic and the dielectric media of Fig. 4(a) is equivalent to the interchange of the positive and the negative directions of the $z$-axis in the Maxwell equations. Therefore, the situations depicted in Fig. 4(a) and (b) are completely equivalent-showing the same dispersion relation.

Then, we consider the case of the nonmagnetic metallic layer surrounded by two identical ferromagnetic dielectrics $(d 1$ and $d 2$ ) assuming that the magnetization in both dielectric media are pointing in the positive direction of the $y$-axis as shown in Fig. 4(c). As we have mentioned before, two independent SPP modes can be excited for thick layers-one in each metal/dielectric interface. Therefore, the mode located at the $d 1 /$ metal interface shows the propagating wave vector $\left(k_{x}\right)$
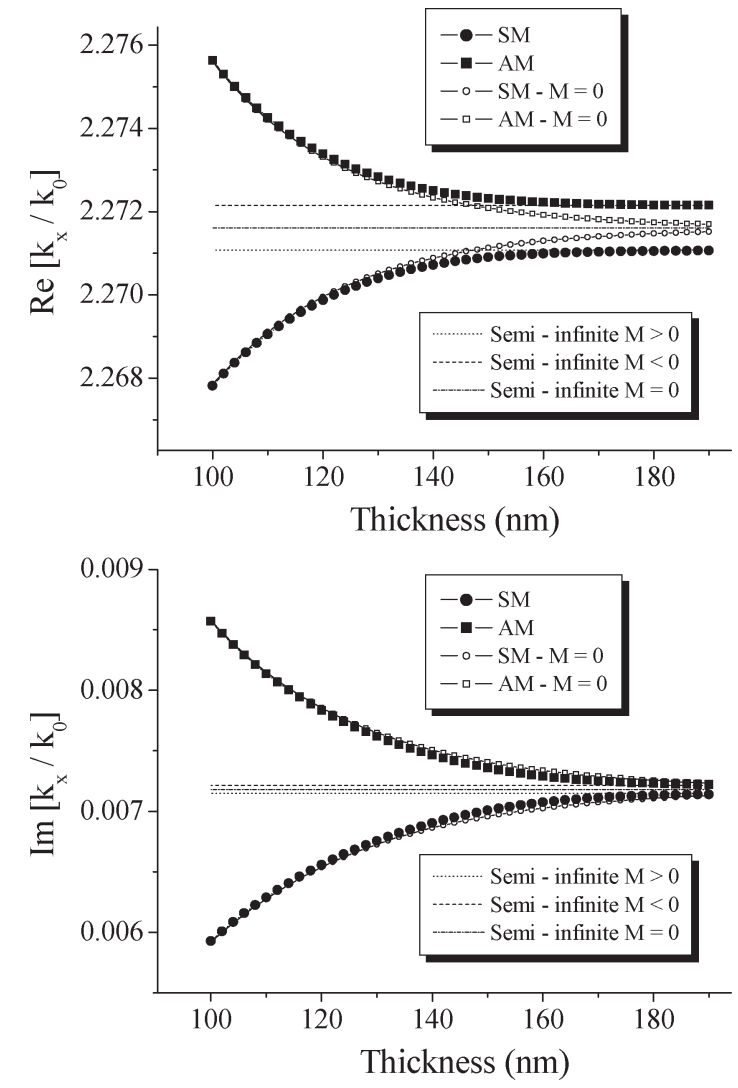

Fig. 5. Real and imaginary parts of the propagation wave vector $k_{x}$ for a gold layer surrounded by YIGs with magnetizations pointing in the positive direction of the $y$-axis as a function of the metal thickness. These solutions are compared with the semi-infinite solutions and the solutions without magnetization. In this configuration, the inversion of the magnetization does not change the wave vector.

corresponding to the solution of (8) for a positive value of the magnetization, whereas the mode located at the $d 2 /$ metal interface corresponds to the negative value of the magnetization. Such a behavior is a consequence of the equivalence of the situations shown in Fig. 4(a) and (b). The reduction of the thickness of the metallic layer and the consequent coupling of the SPP generate the SM and the AM-producing the loss of the nonreciprocal effect. This behavior can be observed in Fig. 5, where the dispersion relation of the SPP for a gold layer surrounded by YIGs with magnetizations parallel to the positive direction of the $y$-axis as a function of the metal thickness is depicted. In these simulations, we have assumed that the diagonal element of the dielectric tensor of the YIG is $\varepsilon_{d}=4.84$, while the nondiagonal MO element is $a M=0.005 i$ [19] for a saturated magnetization.

In these graphs, we represent the thickness dependence of the wave vector of the SPP with and without magnetization in the dielectric layer, together with the solutions for one interface

$$
k_{x}( \pm M)=k_{0} \sqrt{\frac{\varepsilon_{m}\left(\varepsilon_{m} \varepsilon_{d}+\varepsilon_{d}^{2}+\left(a_{d} M\right)^{2} \pm 2 \varepsilon_{m} a_{d} M \sqrt{\frac{\varepsilon_{m} \varepsilon_{d}}{\left(\varepsilon_{m}-\varepsilon_{d}\right)^{2}+\left(a_{d} M\right)^{2}}}\right)}{\left(\varepsilon_{m}+\varepsilon_{d}\right)^{2}+\left(a_{d} M\right)^{2}}}
$$



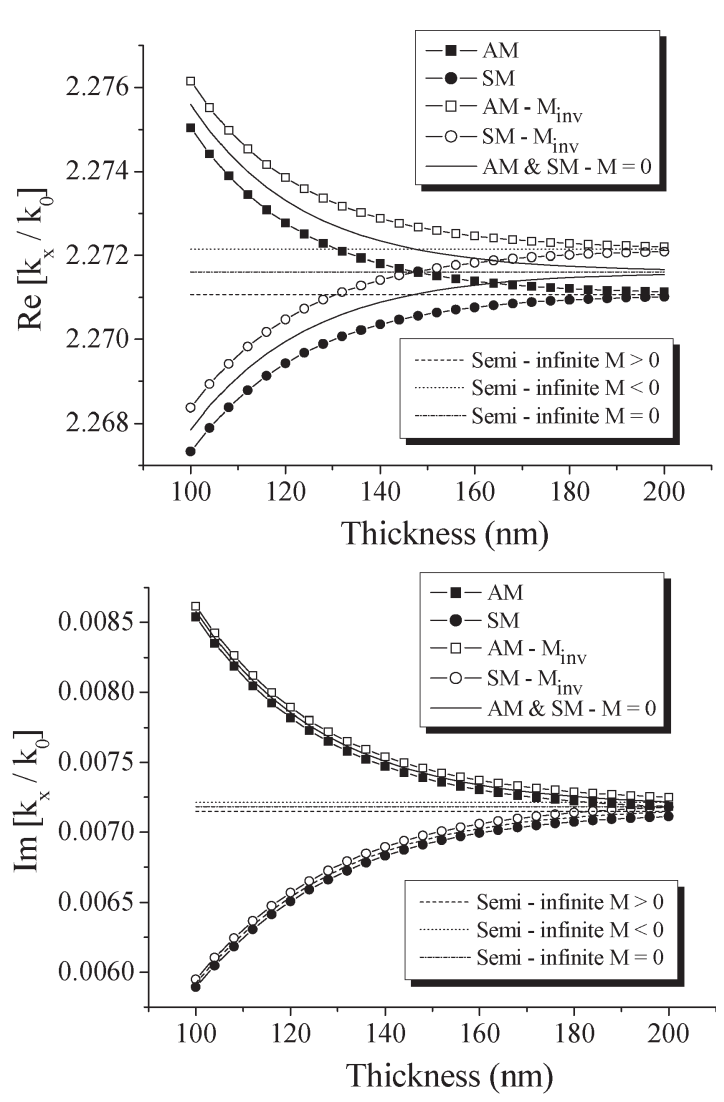

Fig. 6. Real and imaginary parts of the propagation wave vector $k_{x}$ for a gold layer surrounded by YIGs with the magnetizations in opposite directions as a function of the gold thickness. These solutions are compared with the solution with the magnetizations inverted, with the solutions without magnetization, and with the semi-infinite solutions.

given by (1) and (8) as horizontal lines. As can be observed, if the magnetization is not considered, the solutions of the SM and the AM converge to the value given by (1) for thick layers. In this situation, the top and bottom interfaces are equivalent, and the SPP show the same dispersion relation. However, when the magnetization is introduced, the solutions of the SM and the AM converge to the different values of (8). In contrast, the difference between the wave vectors with and without magnetization tends quickly to zero as the metallic thickness is reduced, and the inversion of the magnetization does not change the dispersion relation of the SM and the AM. In conclusion, the interaction between the SPPs in a symmetric configuration of dielectric layers and magnetization produces the cancellation of the nonreciprocal effect.

On the other hand, when the magnetizations of the dielectric media are in opposite directions, as the case in Fig. 4(d), the MO effects are different as can be observed in Fig. 6. In the case of thick layers, the independent SPP modes at the top and bottom interfaces are equivalent and have the same dispersion relation given by one of the values of (8). As a consequence, the SM and the AM tend to this value as the metallic thickness increases, as observed in Fig. 6. The inversion of the magnetization in both layers produces a shift in the wave vector of both SPPs to the other solution of (8). Therefore, when the magnetizations of the dielectric media are in opposite directions, the interaction of the two SPP modes does not cancel the nonreciprocal effect
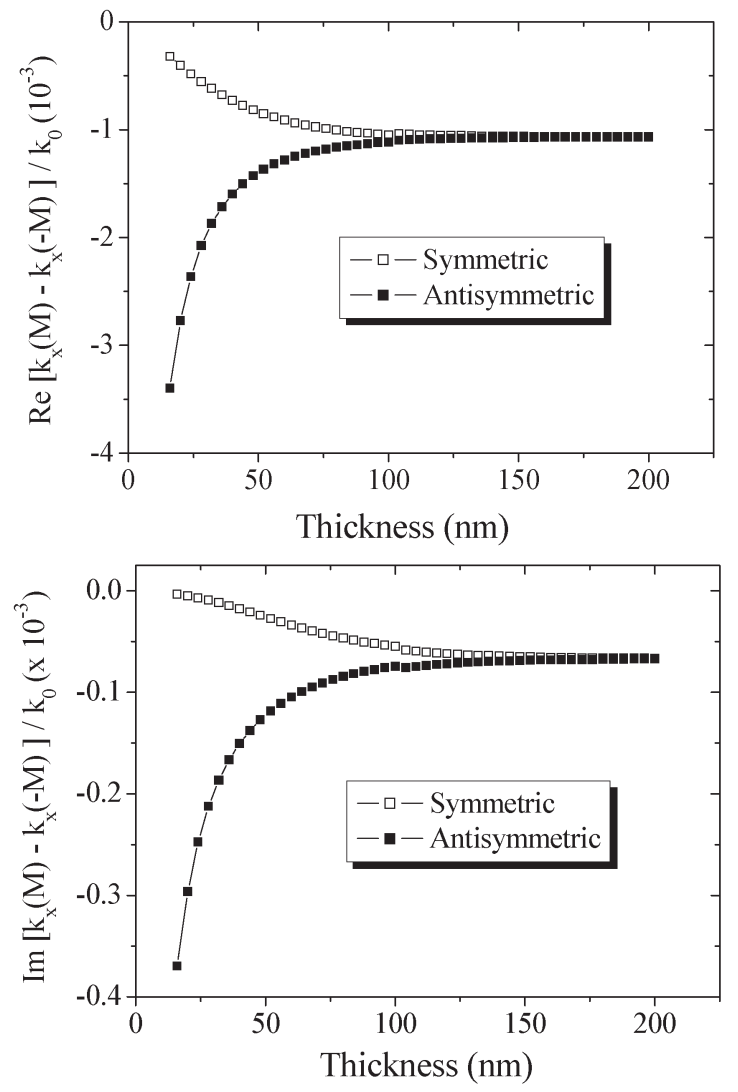

Fig. 7. Difference of $k_{x}$ when the magnetization is inverted $\left[k_{x}(M)-\right.$ $\left.k_{x}(-M)\right]$ for a gold layer surrounded by YIGs with the magnetizations in opposite directions as a function of the gold thickness.

induced by the magnetization (i.e., $k_{x}^{+}(\bar{M}) \neq k_{x}^{+}(-\bar{M})$ for the SM and the AM).

The difference induced in $k_{x}$ by the inversion of the magnetizations of the dielectric layers as a function of the metal thickness is shown in Fig. 7.

As can be observed, the nonreciprocal displacement of $k_{x}$ tends slowly to zero in the SM as the thickness of the metal layer is diminished. Also, Fig. 7 shows that the reduction of the metallic thickness enhances the nonreciprocal MO effect in the AM.

Such a nonreciprocal effect in $k_{x}$ can also be found in an asymmetric structure formed by the metallic layer bounded by a magnetic dielectric material at one side and by a nonmagnetic dielectric with a different dielectric constant at the other. In Fig. 8, we present the same results in Fig. 7 as an example; but in this case, the ferromagnetic dielectric at one of the sides of the metal is substituted by a nonmagnetic dielectric with a higher dielectric constant $\left(\varepsilon_{d 2}=6.25\right)$.

As can be observed, the MO effect of the HIM is negligible for thick layers because the mode is bounded at the interface of the gold layer and the nonmagnetic dielectric medium, and the penetration in the YIG is very small. The reduction of the gold thickness increases the penetration of the HIM into the magnetic dielectric, causing the enhancement of the nonreciprocal shift for thin layers. On the other hand, the MO effect in the LIM is approximately maintained up to the cutoff 

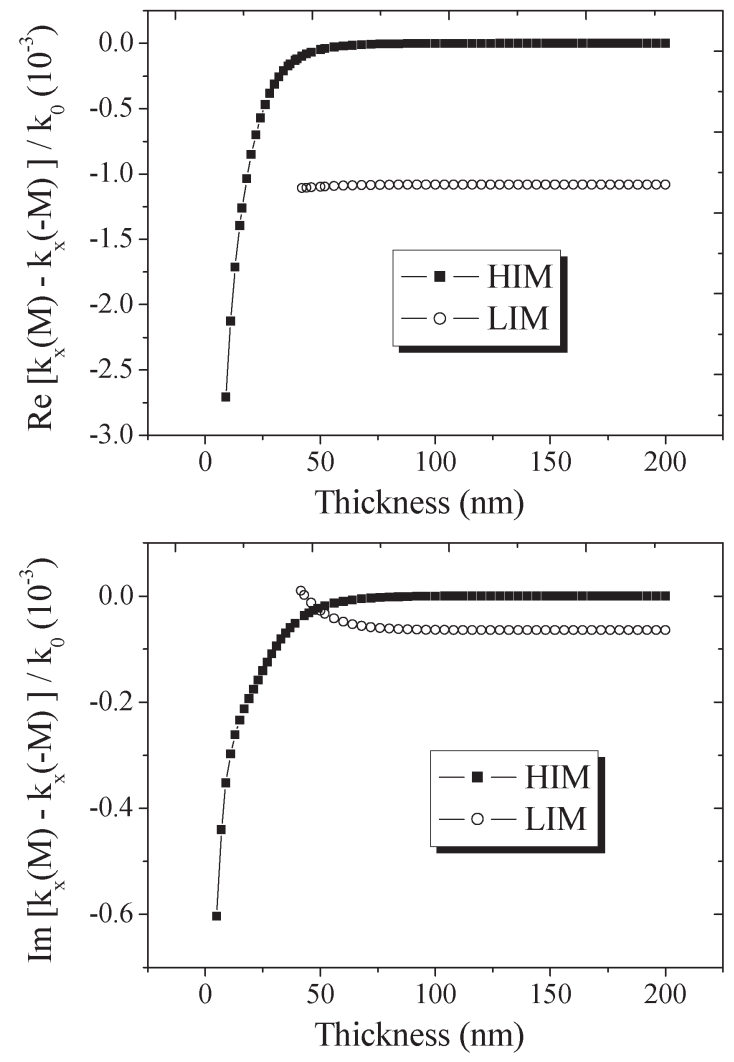

Fig. 8. Difference of $k_{x}$ when the magnetization is inverted $\left[k_{x}(M)-\right.$ $\left.k_{x}(-M)\right]$ for a gold layer surrounded by YIGs at one side and a nonmagnetic dielectric with a higher dielectric constant $\varepsilon_{d 2}=6.25$ at the other side as a function of the gold thickness.

thickness. If the MO dielectric medium has a higher dielectric constant than the nonmagnetic dielectric, then the LIM shows a negligible $\mathrm{MO}$ effect for thick layer; and the effect increases as the thickness diminishes and the mode extends into the MO medium. However, such an increase is more than an order of magnitude weaker than the previous configuration. Regarding the HIM, its MO effect is roughly maintained for thick layers and begins to increase for thin layers, as the field is concentrated at the interface of the gold and the magnetic dielectric medium.

If we consider a ferromagnetic metal layer like Co surrounded by two dielectric media without an MO activity instead of considering a metal without an MO activity surrounded by dielectrics with an MO activity, the MO effects are very similar. The symmetric configuration, in which the ferromagnetic metal is bounded by dielectrics with the same optical constants, is equivalent to the case of a gold layer surrounded by two YIG layers with the magnetization pointing in the same directions. In the same way, the asymmetric configuration with a cobalt layer bounded by dielectric layers with different dielectric constants can be explained like the asymmetric gold configuration. The only difference is found in the shape of the dispersion relations due to the higher absorption of cobalt compared to gold.

In conclusion, in order to obtain nonreciprocal effects induced by the magnetization in a wide range of thickness, the existence of any kind of asymmetry is necessary in the dielectric constants of the SPP waveguide or in the direction of the magnetizations. Although the most confined modes within the metal, which are the ones that show more attenuation, presents the highest MO effects; it is possible to achieve long-range modes while maintaining high MO effects, thus opening the way to technological applications, as will be discussed in the next section.

\section{B. Longitudinal Magnetization}

If the magnetization is in the longitudinal direction, that is, the direction of propagation of the SPP, the dielectric tensor of a magnetic material is given by

$$
\varepsilon=\left(\begin{array}{ccc}
\varepsilon & 0 & 0 \\
0 & \varepsilon & a M \\
0 & -a M & \varepsilon
\end{array}\right)
$$

As a consequence, the magnetization couples the $y$ and the $z$ components of the electric field, impeding the separation of the TE and the TM polarizations. Within this context, only the symmetric configurations are considered, since the asymmetric configuration can be figured out from the behavior of the former.

The coupling induced by the magnetization gives rise to SPP modes that have all the components of the electromagnetic field; and therefore, the guided modes are not purely TM. The dispersion relations of such modes are very similar to the modes without magnetization, since the longitudinal magnetization produces a very weak change in $k_{x}$-this change being reciprocal [28].

In the case of a ferromagnetic layer surrounded by nonmagnetic dielectric media, the electromagnetic field distributions of the SM and the AM modes are displayed in Fig. 9. In this calculation, we have used the dielectric and the MO constants of cobalt at a wavelength of $1500 \mathrm{~nm}$, i.e., $\varepsilon_{m}=-8.2+59.75 i$ and $a M=-1.4858+0.9832 i$ [24], and we have assumed that the dielectric media surrounding the cobalt have a dielectric constant $\varepsilon_{d}=2.13$, which is similar to silicon oxide.

In this figure, the $y$ and the $z$ components of the magnetic fields $\left[H_{y}(z)\right.$ and $\left.H_{z}(z)\right]$ are represented. The $y$ component, which corresponds to the TM part of the mode, exhibits the usual field distribution of the SM and the AM. Thus, the SM tends to extend into the dielectric, while the AM penetrates deeper into the metal as the metal thickness diminishes. The $z$ component created by the magnetization have the same behavior as $H_{y}(z)$ as a function of the metal thickness. However, if the amplitude of $H_{z}(z)$ at the interfaces of the metal is compared with the amplitude of $H_{y}(z)$, an enhancement of the MO effect in the SM can be observed when the metal thickness is reduced. Meanwhile, the $z$ component of the AM is reduced when the metallic thickness is diminished. Such behavior can be computed as the rate $H_{z} / H_{y}$ at the interface of the metal and the dielectric as a function of the thickness of the metal as represented in Fig. 10.

On the other hand, the field distributions of the SPP guided modes when the MO activity is in the dielectric media and the metal is nonmagnetic are presented in Fig. 11. The $H_{y}(z)$ 

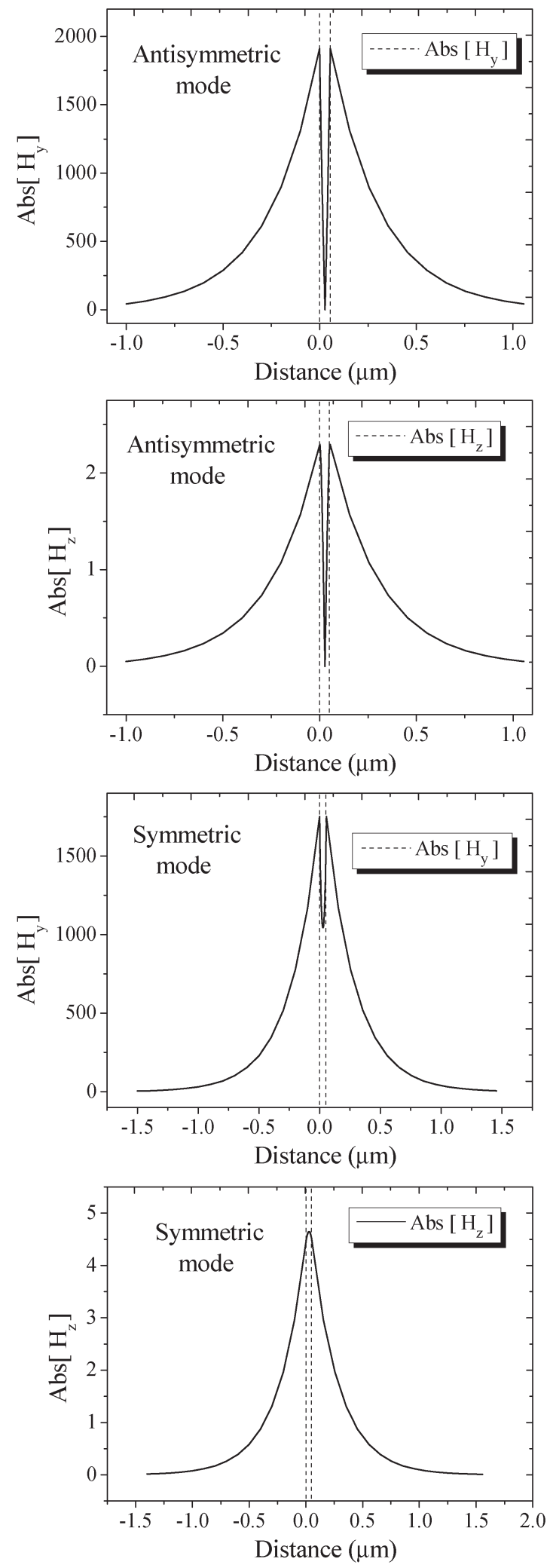

Fig. 9. Field distribution of $H_{y}$ and $H_{z}$ for a cobalt layer of $50 \mathrm{~nm}$ for the $\mathrm{AM}$ and the SM in the longitudinal direction of the magnetization.

component again shows the normal behavior and the field distribution of the SM and the AM; and then, the longitudinal magnetization only produces a little diminution of the field amplitude in this component. However, the nonTM component

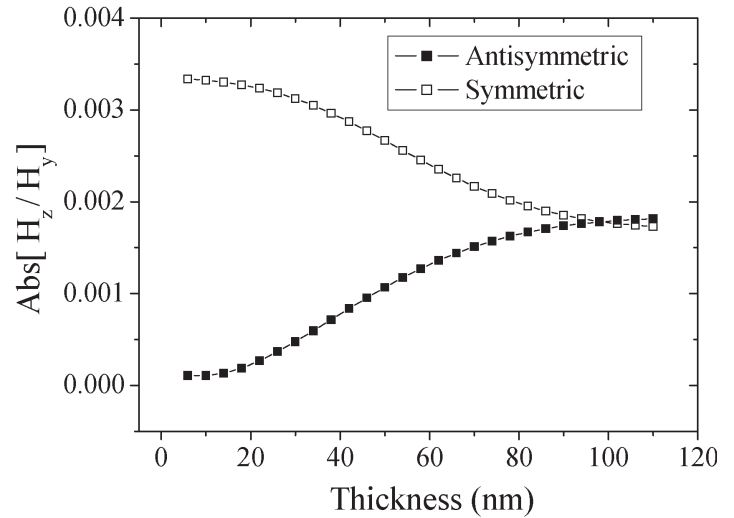

Fig. 10. Rate $H_{y} / H_{z}$ at the interface of the metal and the dielectric as a function of the cobalt thickness.

$H_{z}$ presents a completely different field distribution. Such a component shows a minimum inside the metallic layer and a maximum in each $\mathrm{MO}$ dielectric medium, followed by an exponential decay, as shown in Fig. 11. Similarly, in the case of the ferromagnetic metallic layer, the reduction of the metal thickness enhances the amplitude of the MO component $H_{z}$ in the SM while the amplitude decreases in the AM.

In conclusion, contrary to the transversal configuration, the MO effects in the longitudinal direction of the magnetization are enhanced as the SPP mode is less confined into the metal. Also, this effect is produced as well in the asymmetric configurations of the dielectric layers. In this configuration, the $H_{z}$ component of the LIM increases as the metal layer is reduced down to the cutoff thickness. However, such increase is weaker than the symmetric configuration. The SPP modes in the longitudinal direction of the magnetization have almost the same propagation constant and attenuation as the equivalent mode without magnetization. As a consequence, a metallic layer can guide long-range SPP modes with low attenuation even though they lose their TM character.

Finally, the MO effects when the magnetization lays in the $z$ direction (polar magnetization) are very similar to the effects in the longitudinal magnetization but weaker; and therefore, they will not be treated.

\section{Applications}

As have been seen in the previous section, there are configurations that present high $\mathrm{MO}$ effects and long propagation distances-offering the possibility of an envisaged technological application.

In the transversal direction of the magnetization, the magnitude of an MO effect can be quantified representing the distance necessary to achieve a $\pi / 2$ phase difference $\left(d_{\pi / 2}\right)$ between a forward and a backward traveling modes or, considering (9), the distance to obtain the same phase difference when the magnetization is reversed

$$
\begin{aligned}
d_{\frac{\pi}{2}} & =\frac{\pi}{2 \operatorname{Re}\left[k_{x}^{+}(M)-k_{x}^{-}(M)\right]} \\
& =\frac{\pi}{2 k_{0} \operatorname{Re}\left[k_{x}^{+}(M)-k_{x}^{+}(-M)\right]} .
\end{aligned}
$$


This distance should be compared to the propagation distance of the mode $\left(d_{\text {prop }}\right)$, which is given by

$$
d_{\text {prop }}=\frac{1}{2 \operatorname{Im}\left[k_{x}\right]} .
$$

The transversal $\mathrm{MO}$ effect poses a technological interest as soon as $d_{\text {prop }}>d_{\pi / 2}$. In this way, we found two interesting arrangements in Section III. The first one is formed by a gold layer surrounded by YIGs at both sides but with magnetizations in opposite directions.

In such an arrangement, although the nonreciprocal phase difference diminishes with the metallic film thickness, the reduction of the attenuation loss is more important as can be observed in Fig. 12(a). As a result, $d_{\text {prop }}>d_{\pi / 2}$ is achieved below $20 \mathrm{~nm}$.

This configuration of the magnetizations can be obtained if the YIGs at both sides show different coercitive magnetic fields. In this case, there will be a range of fields in which the orientations of the magnetizations are antiparallel. Another possibility could be found if one of the YIGs is coupled to a hard magnetic layer with a high coercitivity. In both cases, the external magnetic fields required will be low and can be obtained with coils or permanent magnets.

Regarding the second arrangement, it is constituted by a gold layer bounded at one side by a YIG and by a nonmagnetic dielectric medium with a higher dielectric constant at the other side. Then, the quantity of electromagnetic field in the MO layer is approximately maintained while the fields stretch out into the higher index layer as the metallic thickness is reduced. As a consequence, the MO effect is conserved and diminishes strongly the attenuation near the cutoff thickness, as shown Fig. 12(b). Then, $d_{\text {prop }}>d_{\pi / 2}$ is reached in the proximity of the cutoff thickness.

Therefore, in both arrangements, there are some film thicknesses where $d_{\text {prop }}>d_{\pi / 2}$, which offer the opportunity to envisage an active MO device based on SPPs (for example, an isolator or a switch). The device could be similar to the nonreciprocal Mach-Zehnder interferometer developed for conventional optical waveguides [29] - replacing the optical waveguides by, for instance, thin gold stripes. If the interferometer is asymmetric with a $\pi / 2$ phase difference between the branches, a nonreciprocal $\pi / 2$ phase difference will produce a constructive interference in a forward propagating mode and a destructive interference for a backward mode. Also, the inversion of the magnetization will produce the destructive interference of the forward mode, which gives rise to an $\mathrm{MO}$ SPP switch. This configuration of an isolator or a switch based on plasmonic waveguides can show some advantages compared to the same devices based on conventional integrated optics as the possibility to generate the magnetic field required to control the magnetization of the dielectric layers passing current through the metal stripes. However, in the case of the asymmetric configuration, the difficulty of working with an SPP mode close to the cutoff must be taken into account. In such a configuration, the roughness of the layers or the small defects in the fabrication can produce the radiation of the LIM mode
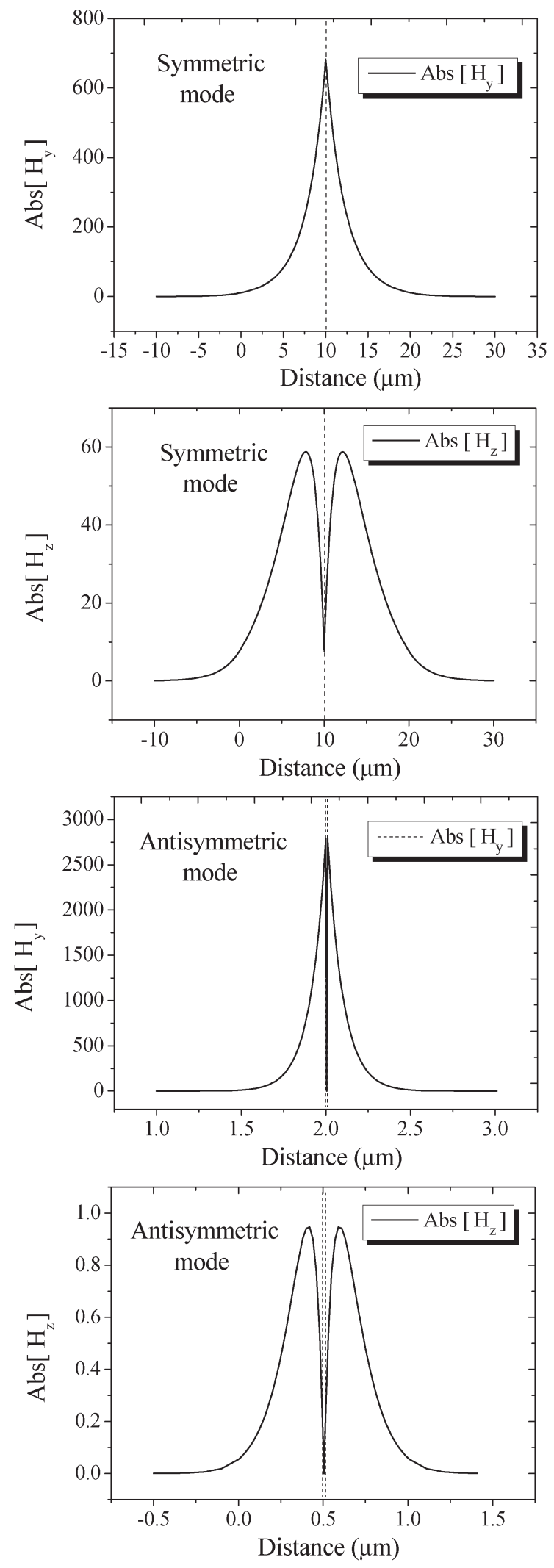

Fig. 11. Field distribution of $H_{y}$ and $H_{z}$ for a gold layer of $10 \mathrm{~nm}$ for the $\mathrm{AM}$ and the SM in the longitudinal direction of the magnetization.

into the dielectric layer with a higher dielectric constantinducing a strong diminution of the propagation distance of the mode. 

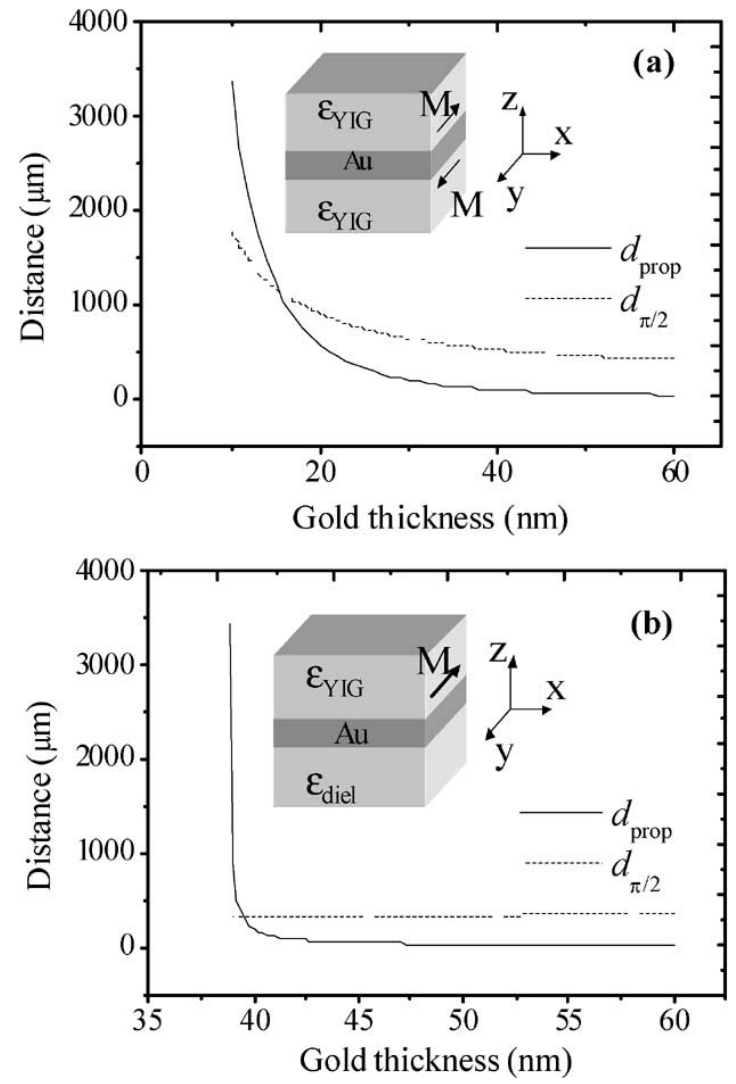

Fig. 12. Distance necessary to obtain a $\pi / 2$ phase shift in the transversal configuration compared to the propagation distance of the SPP as a function of the metallic thickness. (a) Metallic layer is surrounded by the same MO medium with magnetizations in opposite directions. (b) Metallic layer presents an MO dielectric medium at one side and a non-MO medium with a higher refractive index at the other side.

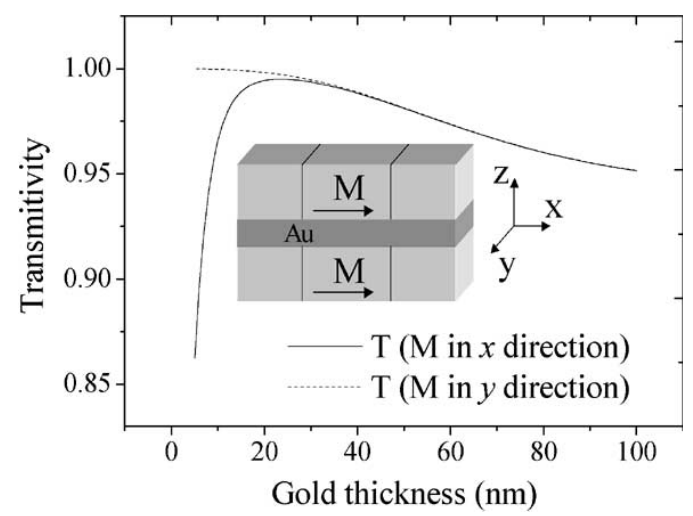

Fig. 13. Transmitivity of the SPP through the structure shown in the inset as a function of the metallic thickness when the magnetization is in the $x$ direction or in the $y$ direction.

Finally, the results obtained in the longitudinal direction of the magnetization suggest considering the propagation through the guiding structure of the inset of Fig. 13-where the SPP mode travels in a nonmagnetic metallic layer surrounded by isotropic media, passes through a waveguide with MO activity in the dielectric media and the magnetization in the direction of propagation, and again passes to an isotropic media. At every step discontinuity, there will be radiation losses [30], [31] of which the intensity will depend on the amplitude of TE compo- nents of the SPP mode. In Fig. 13, we present the transmitivity of the mode through such a structure, which has been calculated using a matrix formalism based on three points [32].

1) The general orthogonality relation obtained from the reciprocity theorem [28]

$$
\begin{aligned}
\left(k_{x \nu}^{\prime}+k_{x n}\right) \iint\left(E_{T \nu}^{\prime}(y, z) \times H_{T n}(y, z)\right. & \\
& \left.-E_{T n}(y, z) \times H_{T \nu}^{\prime}(y, z)\right) \cdot u_{x} d y d z=0
\end{aligned}
$$

where $E_{T n}$ and $H_{T n}$ are the transversal components of the electric and magnetic fields of the SPP mode $n$ of the metallic layer, respectively. On the other hand, the prime mode represents the SPP mode $\nu$ of the guiding structure in a situation in which the dielectric tensors of the MO media are transposed, i.e., with the magnetizations inverted. In such a situation, the metallic waveguide is denoted as the complementary waveguide. In (13), $k_{x n}$ and $k_{x \nu}^{\prime}$ are the wave vectors of the SPP modes $n$ and $\nu$ of the normal and the complementary waveguides, respectively, and $u_{x}$ represents the unitary vector in the $x$ direction.

2) The modal expansion of the transverse electromagnetic fields within the metallic waveguide

$$
\begin{aligned}
\mathcal{E}_{T}=\sum_{n=0}^{M}( & a_{n} \cdot E_{T n}^{+}(y, z) e^{i k_{x n}^{+} x} \\
& \left.+b_{n} \cdot E_{T n}^{-}(y, z) e^{i k_{x n}^{-} x}\right)+E_{\mathrm{rad}} \\
\mathcal{H}_{T}=\sum_{n=0}^{M}( & a_{n} \cdot H_{T n}^{+}(y, z) e^{i k_{x n}^{+} x} \\
& \left.+b_{n} \cdot H_{T n}^{-}(y, z) e^{i k_{x n}^{-} x}\right)+H_{\mathrm{rad}}
\end{aligned}
$$

where $\left\{E_{T n}^{+}, H_{T n}^{+}\right\}$and $\left\{E_{T n}^{-}, H_{T n}^{-}\right\}$are the transverse components of the electromagnetic fields of the nonradiative modes in the forward and backward directions of propagation, respectively, while $E_{\mathrm{rad}}$ and $H_{\mathrm{rad}}$ represent the radiative modes of the metallic guiding structure.

3) The continuity of the tangential components of such electromagnetic fields at both sides of the discontinuity $\left(E_{y}, E_{z}, H_{y}\right.$, and $\left.H_{z}\right)$.

With these expressions, the electromagnetic fields at both sides of the MO metallic waveguide of Fig. 13 can be related, and this relation can be expressed in the matrix form [32]. With these relations, the transmitivity and the reflectivity of the SPP modes can be calculated. In the calculations, we have first assumed that the MO waveguide has a length of $1 \mu \mathrm{m}$, that all the dielectric media have the same diagonal dielectric constants, and that the metallic thickness is maintained in the whole structure. The optical and MO parameters are those of YIG and gold for $1.5 \mu \mathrm{m}$. Second, we assume that only the SM arrives at the interface of the first isotropic waveguide; and finally, we neglect the coupling of the radiative modes at the discontinuities. This assumption can be considered since the discontinuities in the dielectric constants are very weak. 
For thick layers, the SPP mode shows strong attenuation since the electromagnetic field is more localized in the metal region and, as a consequence, the TE components of this mode induced by the $\mathrm{MO}$ activity of the dielectric layers are small. However, for thinner layers (below $20 \mathrm{~nm}$ ), the electromagnetic field spreads into the dielectric layers while the TE components of the SPP mode increase-inducing an MO effect that begins to be more important than the attenuation of the metallic layer. Such a behavior produces a reduction of the transmitivity due to the radiation losses at the discontinuities, as shown in Fig. 13.

If the magnetization of the structure is rotated up to the transversal configuration, the radiation losses disappear because, in this configuration, the SPP mode recovers its TM character (Fig. 13). Therefore, a rotating magnetization within the $x y$ plane will induce a modulation of the transmitivity of the SPP - an effect that can be enhanced by the addition of more discontinuities. Moreover, this rotation allows the control of the emission of the radiation at the discontinuities.

\section{CONClusion}

In this paper, we have presented the MO effects in SPP modes guided by metal layers as a function of the metallic thickness for different orientations of the magnetization.

We have shown within the transversal direction of the magnetization that, when the configuration of the dielectric media and the magnetizations are symmetric, the nonreciprocal MO effects produced at a single interface are cancelled by the interaction of the SPP at both interfaces of the metallic layer. Then, to obtain a nonreciprocal effect, the existence of any asymmetry between the dielectric layers is necessary, either in the dielectric constant or in the magnetization. Moreover, the MO effect is enhanced as the SPP modes are more confined into the metal and tends to zero as the modes converge to the solution of a plane wave. However, it is possible to find longrange modes while maintaining high nonreciprocal shifts in the dispersion relation.

Regarding the longitudinal direction of magnetization, we have demonstrated that non-TM modes can be guided by a metallic layer with almost same dispersion relation and propagation losses as the guiding structure without magnetization. In this case, the non-TM components of the electromagnetic field are enhanced as the modes are less bounded to the metal.

Finally, we have shown that strong MO effects can be obtained for the long-range modes. Different configurations have been analyzed, which show the possibility to design new active optical devices based on SPPs.

\section{REFERENCES}

[1] W. L. Barnes, A. Dereux, and T. W. Ebbesen, "Surface plasmon subwavelength optics," Nature, vol. 424, no. 6950, pp. 824-830, Aug. 2003.

[2] P. Berini, "Plasmon-polariton waves guided by thin lossy metal films of finite width: Bound modes of symmetric structures," Phys. Rev. B, Condens. Matter, vol. 61, no. 15, p. 10484, Apr. 2000.

[3] _ _ "Plasmon-polariton waves guided by thin lossy metal films of finite width: bound modes of asymmetric structures," Phys. Rev. B, Condens. Matter, vol. 63, no. 12, p. 125417, Mar. 2001.

[4] R. Charbonneau, P. Berini, E. Berolo, and E. Lisicka-Shrzek, "Experimental observation of plasmon-polariton waves supported by a thin metal film of finite width," Opt. Lett., vol. 25, no. 11, pp. 844-846, Jun. 2000.

[5] B. Lamprecht, J. R. Krenn, G. Schider, H. Ditlbacher, M. Salerno, N. Felidj, A. Leitner, F. R. Aussenegg, and J.-C. Weeber, "Surface plasmon propagation in microscale metal stripes," Appl. Phys. Lett., vol. 79, no. 1, pp. 51-53, Jul. 2001.

[6] J.-C. Weeber, J. R. Krenn, A. Dereux, B. Lamprecht, Y. Lacroute, and J. P. Goudonnet, "Near-field observation of surface plasmon polariton propagation on thin metal stripes," Phys. Rev. B, Condens. Matter, vol. 64, no. 4, p. 045411, Jul. 2001.

[7] J.-C. Weeber, Y. Lacroute, and A. Dereux, "Optical near-field distributions of surface plasmon waveguide modes," Phys. Rev. B, Condens. Matter, vol. 68 , no. 11, p. 115401, Sep. 2003.

[8] H. Ditlbacher, J. R. Krenn, G. Schider, A. Leitner, and F. R. Aussenegg, "Two-dimensional optics with surface plasmon polaritons," Appl. Phys. Lett., vol. 81, no. 10, pp. 1762-1764, Sep. 2002.

[9] S. I. Bozhevolnyi, V. S. Volkov, K. Leosson, and A. Boltasseva, "Bend loss in surface plasmon polariton band-gap structures," Appl. Phys. Lett., vol. 79, no. 8, pp. 1076-1078, Aug. 2001.

[10] R. F. Wallis, J. J. Brion, E. Burstein, and A. Hartstein, "Theory of surface polaritons in anisotropic dielectric media with application to surface magnetoplasmons in semiconductors," Phys. Rev. B, Condens. Matter, vol. 9, no. 8, p. 3424, Apr. 1974.

[11] R. L. Stamps, B. L. Johnson, and R. E. Camley, "Nonreciprocal reflection from semi-infinite antiferromagnets," Phys. Rev. B, Condens. Matter, vol. 43, no. 4, p. 3626, Feb. 1991.

[12] M. S. Kushwaha and P. Halevi, "Magnetoplasma modes in thin-films in the Faraday configuration," Phys. Rev. B, Condens. Matter, vol. 35, no. 8, pp. 3879-3889, Mar. 1987.

[13] Rev. B, Condens. Matter, vol. 36, no. 11, pp. 5960-5967, Oct. 1987.

[14] _ - "Magnetoplasmons in thin-films in the perpendicular configuration," Phys. Rev. B, Condens. Matter, vol. 38, no. 17, pp. 12428-12435, Dec. 1988.

[15] P. E. Ferguson, O. M. Stafsudd, and R. F. Wallis, "Enhancement of transverse Kerr magneto-optic effect by surface magnetoplasma waves," Physica B and C, vol. 86, no. JAN-M, pp. 1403-1405, 1977.

[16] N. Richard, A. Dereux, T. David, E. Bourillot, J. P. Goudonnet, F. Scheurer, E. Beaurepaire, and G. Garreau, "Magneto-optical effects in multilayers illuminated by total internal reflection," Phys. Rev. B, Condens. Matter, vol. 59, no. 8, p. 5936, Feb. 1999.

[17] C. Hermann, V. A. Kosobukin, G. Lampel, J. Peretti, V. I. Safarov, and P. Bertrand, "Surface-enhanced magneto-optics in metallic multilayer films," Phys. Rev. B, Condens. Matter, vol. 64, no. 23, p. 235422, Dec. 2001.

[18] Y. M. Strelniker and D. J. Bergman, "Optical transmission through metal films with a subwavelength hole array in the presence of a magnetic field," Phys. Rev. B, Condens. Matter, vol. 59, no. 20, pp. R12763-R12766, May 1999.

[19] T. Shintaku and T. Uno, "Preparation of Ce-substituted yttrium iron garnet films for magneto-optic waveguide devices," Jpn. J. Appl. Phys., vol. 35, no. 9A, pp. 4689-4691, Sep. 1996.

[20] A. D. Boardman, Electromagnetic Surface Modes. Chichester, U.K.: Wiley, 1982, pp. 16-17.

[21] E. N. Economou, "Surface plasmons in thin films," Phys. Rev., vol. 182, no. 2 , p. $539,1969$.

[22] J. J. Burke, G. I. Stegeman, and T. Tamir, "Surface-polariton-like waves guided by thin, lossy metal-films," Phys. Rev. B, Condens. Matter, vol. 33, no. 8, pp. 5186-5201, Apr. 1986.

[23] B. Prade, J. Y. Vinet, and A. Mysyrowicz, "Guided optical waves in planar heterostructures with negative dielectric-constant," Phys. Rev. B, Condens. Matter, vol. 44, no. 24, pp. 13556-13572, Dec. 1991.

[24] J. H. Weaver, C. Krafka, D. W. Lynch, and E. E. Kock, Physics Data: Optical Properties of Metals. Karlsruhe, Germany: Fachinformationszentrum Karlsruhe, 1981.

[25] M. O. Vassell, "Structure of optical guided modes in planar multilayers of optically anisotropic materials," J. Opt. Soc. Amer., vol. 64, no. 2, pp. 166-173, 1974.

[26] M. Schubert, "Polarization-dependent optical parameters of arbitrarily anisotropic homogeneous layered systems," Phys. Rev. B, Condens. Matter, vol. 53, no. 8, p. 4256, Feb. 1996.

[27] P. Berini and K. Wu, "Modeling lossy anisotropic dielectric waveguides with the method of lines," IEEE Trans. Microw. Theory Tech., vol. 44, no. 5, pp. 749-759, May 1996.

[28] P. R. McIsaac, "Mode orthogonality in reciprocal and nonreciprocal wave-guides," IEEE Trans. Microw. Theory Tech., vol. 38, no. 11, pp. 1808-1816, Nov. 1991. 
[29] J. Fujita, M. Levy, R. M. Osgood, L. Wilkens, and H. Dötsch, "Waveguide optical isolator based on Mach-Zehnder interferometer," Appl. Phys. Lett., vol. 76, no. 16, pp. 2158-2160, Apr. 2000.

[30] J. A. Sánchez-Gil, "Surface defect scattering of surface plasmon polaritons: Mirrors and light emitters," Appl. Phys. Lett., vol. 73, no. 24, pp. 3509-3511, Dec. 1998.

[31] J. A. Sánchez-Gil and A. A. Maradudin, "Near-field and far-field scattering of surface plasmon polaritons by one-dimensional surface defects," Phys. Rev. B, Condens. Matter, vol. 60, no. 11, pp. 8359-8367, Sep. 1999.

[32] B. Sepúlveda and L. M. Lechuga, "Matrix analysis of discontinuities in nonreciprocal waveguides: Analytical description for magnetooptical slab waveguides," J. Lightw. Technol., vol. 22, no. 7, pp. 1772-1781, Jul. 2004.

B. Sepúlveda received the Ph.D. degree in physics from the University Complutense, Madrid, Spain, in 2005.

He currently has a two year postdoctoral position with the Nanoplasmonic Group, Chalmers University of Technology, Gothenburg, Sweden. His research activities are related to integrated optics and plasmonics and their applications in the biosensing and micro/nano optics fields.
L. M. Lechuga received the Ph.D. degree in chemistry from the University Complutense, Madrid, Spain, in 1992.

Since 1995, she has been a Research Staff member of the CNM-CSIC, Madrid. She is now the Head of the Biosensors Group at the Microelectronics National Center, CSIC. The Biosensors Group develops optoelectronics and nanomechanics biosensor at the micro/nano level for integration in microsystem platforms. Her main research areas are the technological development of integrated optical and nanomechanical devices based mainly on IC Silicon micro and nanotechnologies (Interferometers, plasmonics, microcantilevers, and magnetoplasmonics), microfluidics for biosensing, and surface biofuncionalization by nanometer scale techniques.

G. Armelles, photograph and biography not available at the time of publication. 